\title{
Electrokinetic Motion of a Deformable Particle: Dielectrophoretic Effect
}

\author{
${\text { Ye } \mathrm{Ai}^{\mathrm{a}} \text {, Benjamin Mauroy }}^{\mathrm{b}}$, Ashutosh Sharma $^{\mathrm{c}, \mathrm{d}}$ and Shizhi Qian ${ }^{\mathrm{a}} \mathrm{d}^{*}$ \\ ${ }^{a}$ Department of Mechanical and Aerospace Engineering, Old Dominion University \\ Norfolk, VA 23529, USA \\ baboratoire MSC, Université Paris 7 / CNRS, Paris France and Laboratoire J.A. Dieudonné, \\ Université de Nice Sophia-Antipolis / CNRS, Nice, France \\ ${ }^{c}$ Department of Chemical Engineering, Indian Institute of Technology at Kanpur, \\ Kanpur 208016, India \\ ${ }^{\mathrm{d}}$ School of Mechanical Engineering, Yeungnam University \\ Gyongsan 712-749, South Korea
}

Keywords: Dielectrophoresis; Electrophoresis; Fluid-structure interaction; Microfluidics; Particle deformation

\footnotetext{
${ }^{*}$ Correspondence: Professor Shizhi Qian, Department of Mechanical and Aerospace Engineering, Old Dominion University, ECSB 1309, 4700 Elkhorn Avenue, Norfolk, VA 23529-0247, USA

Email: sqian@odu.edu

Fax: +1-757-683-3200
}

Abbreviations: ALE, Arbitrary Lagrangian-Eulerian; DEP, Dielectrophoretic; EDL, Electrical Double Layer; EOF, Electroosmotic Flow; RBC, Red Blood Cell; 2D, Two-dimensional 


\begin{abstract}
Electrokinetics-induced motion and deformation of a hyperelastic particle confined in a slit microchannel has been numerically investigated for the first time with a full consideration of the fluid-particle-electric field interactions and the dielectrophoretic (DEP) effect. When the initial orientation of a cylindrical particle with respect to the applied electric field, $\theta_{p 0}$, is $90^{\circ}$, the particle tends to curl up as a "C" shape when moving from left to right. The electrokineticsinduced particle deformation is due to the joint effects of the shear force arising from the nonuniform Smoluchowski slip velocity on the particle surface, and the asymmetric DEP force with respect to the center of the deformed particle arising from the spatially non-uniform electric field surrounding the particle. The electrokinetics-induced particle deformation is opposite to that of a particle moving in the same direction subjected to a pressure-driven flow. When the initial particle orientation is $0<\theta_{p 0}<90^{\circ}$, a net torque arising from the DEP effect progressively rotates and aligns the particle with its longest axis parallel to the applied electric field, thus decreasing the non-uniformity of the electric field and accordingly the particle deformation. The numerical predictions are in qualitative agreement with our previous experimental observation. The results show that the DEP effect is significant and must be taken into account in the modeling of electrokinetic motion of a deformable particle in microfluidics.
\end{abstract}




\section{Introduction}

Electrokinetic particle motion refers to the migration of charged particles suspended in aqueous solutions subjected to externally applied electric fields. It is derived from the interplay between the applied electric field and the net charges on the particle surface and within the electrical double layer (EDL) formed adjacent to the charged surface. The electrokinetic phenomena have been widely used to transport and manipulate colloidal particles and biological cells in lab-on-a-chip devices using electric fields [1-3]. Extensive theoretical analyses [4-12] and experimental studies [13-20] have been performed on the electrokinetic motion of rigid particles in micro/nanofluidics.

Recently, there has been a growing interest in studying the deformation of soft particles in microfluidics. Risso et al. [21] experimentally studied the deformation of a bio-artificial capsule subjected to a pressure-driven flow in a confined channel. It has been also known that the deformability of red blood cell (RBC) is associated with its health status [22]. Abkarian et al. [23-24] experimentally observed the alterations of deformability between healthy and unhealthy $\mathrm{RBC}$ in a microchannel. Later, the deformation of RBC subjected to pressure-driven flows in a microchannel has been comprehensively studied [25-27]. Recently, Chen et al. [28] fabricated a lab-on-a-chip device with a capillary network to study the RBC hydrodynamics. All of the above efforts aim to develop a practical lab-on-a-chip device capable of RBC deformability diagnosis in clinical applications. In addition to the rapidly increasing experimental studies on the deformation of soft biological particles, greater efforts have also been made on the development of modeling tools to simulate particle deformation subject to pressure-driven or shear-driven flows [29-34]. Up till now, very little attention has been paid to the particle deformation in electrokinetics-based microfluidic devices. Recently, van den Heuvel et al. [35] experimentally studied electrophoretic motions of semi-flexible rod-like microtubules in a microfluidic channel, and found that microtubules are either aligned to the electric field imposed or deformed into Ushapes when they are perpendicular to the electric field. In addition, the deformed microtubules relax to its initially undeformed shape when the imposed electric field is turned off, which confirms that the electrokinetic effect is responsible for the deformation of the flexible microtubules. Swaminathan et al. [36] numerically studied the electrokinetics-induced deformation of a long elastic particle suspended in an unbounded medium. Confinement of the particle in a microchannel, as well as the dielectrophoretic (DEP) effect arising from the interaction between the dielectric particle and the spatially non-uniform electric field, were neglected in the aforementioned study. However, our previous studies clearly revealed the important role played by the DEP effect in the electrokinetic motion of rigid particles in microfluidics [37-42].

In this paper, a numerical model is developed to simultaneously solve the electric field, hydrodynamic field, and dynamics of a deformable particle using an arbitrary LagrangianEulerian (ALE) method, which is regarded as one of the most efficient computational approaches to deal with moving boundaries in the computational domain [43-44]. The proposed numerical model without considering the particle deformation has already been successfully implemented to simulate the pressure-driven [45] and electrokinetic motion [37-41] of rigid particles in microchannels, indicating good agreements with experimental results. In contrast to the previous study by Swaminathan et al. [36], we investigate for the first time the DEP effect on the transient electrokinetic motion of a deformable particle in a confined microchannel with emphasis on the electrokinetics-induced particle deformation. 


\section{Mathematical Model}

Consider a two-dimensional (2D) incompressible hyperelastic particle $\Omega_{p}$ with homogeneous properties suspended in an incompressible Newtonian fluid domain $\Omega_{f}$ with density $\rho_{f}$, dynamic viscosity $\mu$, and permittivity $\varepsilon_{f}$, which is further confined by two parallel rigid walls with a distance of $d$, as shown in Figure 1. The dielectric cylindrical particle of length $L_{p}$ is capped with two hemispheres of radius $a$ at both ends. Initially, the center of mass of the particle is located at $\left(x_{p 0}, y_{p 0}\right)$, and the longest axis of the particle presents an angle $\theta_{p 0}$ counterclockwise with respect to the centerline of the microchannel. A potential difference is externally applied across both ends of the fluid domain, generating an electric field, $\mathbf{E}$, and simultaneously inducing electrokinetic particle motion inside the microchannel.

The thickness of the EDL formed adjacent to the charged surface is on the order of several nanometers, which is much smaller than the particle radius and the width of the microchannel. As a result, a thin EDL approximation is adopted in the present study, which renders a zero net charge density in the fluid domain $\Omega_{f}[4]$. The good agreements between the experimental results and numerical predictions on electrokinetic motion of rigid particles in microchannels using the thin EDL approximation [37-41] further confirm that the thin EDL approximation is appropriate in many microfluidic applications. Liu et al [51] used three different models: the Smoluchowski's slip velocity (the thin EDL approximation model without considering the EDL), the Poisson-Boltzmann model (considering equilibrium EDL), and the multi-ion model (accounting for EDL polarization), to investigate a cylindrical nanoparticle electrophoretic translation through a nanopore, and found that the predictions of the three models are in good agreement when the EDL is thin. Therefore, the thin EDL approximation is appropriate for the conditions described in this paper.

Under thin EDL approximation, the electrical potential $\phi$ satisfies the Laplace equation

$$
\nabla^{2} \phi=0 \quad \text { in } \Omega_{f} .
$$

The potential difference between the inlet and the outlet is imposed as

$$
\phi=0 \quad \text { on } \mathrm{AB},
$$

and

$$
\phi=\phi_{0} \quad \text { on CD . }
$$

All the other boundaries are electrically insulating,

$$
\mathbf{n}_{f} \bullet \nabla \phi=0 \quad \text { on } \mathrm{BC}, \mathrm{AD} \text {, and } \Gamma \text {, }
$$

where $\mathbf{n}_{f}$ is the unit normal vector directed from the corresponding boundary into the fluid in the 2D spatial frame $(x, y)$ with its origin fixed at the center of the microchannel.

As the Reynolds number of the electrokinetic flow in microfluidics is very small (i.e., typically less than 0.01), the inertia terms in the Navier-Stokes equations are neglected and the fluid motion is modeled by the continuity equation and the Stokes equations, given as

$$
\nabla \bullet \mathbf{u}=0 \quad \text { in } \Omega_{f},
$$

and

$$
\rho_{f} \frac{\partial \mathbf{u}}{\partial t}=-\nabla p+\mu \nabla^{2} \mathbf{u} \quad \text { in } \Omega_{f},
$$

where $\mathbf{u}$ is the fluid velocity vector and $p$ is the pressure.

A normal flow with zero pressure is specified at the two openings of the fluid domain $\Omega_{f}$. If the rigid walls are charged, an electroosmotic flow (EOF) is generated next to the charged 
boundary, which is approximated by the Smoluchowski slip velocity in the present study owing to the thin EDL. As a result, the EOF fluid velocity on the rigid walls is described as

$$
\mathbf{u}=\frac{\varepsilon_{f} \zeta_{w}}{\mu}(\mathbf{I}-\mathbf{n n}) \bullet \nabla \phi \quad \text { on } \mathrm{BC} \text { and } \mathrm{AD},
$$

where $\zeta_{w}$ is the zeta potential of the rigid wall and $\mathbf{I}$ is the second-order unit tensor. The quantity $(\mathbf{I}-\mathbf{n n}) \bullet \nabla \phi$ defines the electric field tangential to the charged surface. The fluid velocity on the particle surface consists of the Smoluchowski slip velocity arising from the particle's surface charge and the velocity related to the particle movement, given as

$$
\mathbf{u}=\frac{\varepsilon_{f} \zeta_{p}}{\mu}(\mathbf{I}-\mathbf{n n}) \bullet \nabla \phi+\frac{\partial \mathbf{w}}{\partial t} \quad \text { on } \Gamma .
$$

In the above, $\zeta_{p}$ is the zeta potential of the particle, and $\mathbf{w}$ is the displacement of the deformable particle which is governed by

$$
\rho_{p} \frac{\partial^{2} \mathbf{w}}{\partial t^{2}}-\nabla \bullet(\boldsymbol{\sigma}(\mathbf{w}))=0 \quad \text { in } \Omega_{p},
$$

where $\rho_{p}$ is the density of the deformable particle and $\boldsymbol{\sigma}(\mathbf{w})$ is the Cauchy stress of the solid phase as a function of the displacement.

We consider the hyperelastic particle as an incompressible Neo-Hookean material, which is described using the following strain energy density function $[34,46]$

$$
W=\frac{G_{0}}{2}\left(I_{C}-3\right) \text {. }
$$

In the above, $G_{0}$ is the shear modulus of the hyperelastic particle and $I_{C}=\operatorname{tr}(\mathbf{C})$ is the first invariant of the right Cauchy-Green tensor, $\mathbf{C}=\mathbf{F}^{T} \mathbf{F}$, where $\mathbf{F}=\nabla_{\mathbf{X}} \mathbf{w}+\mathbf{I}$ is the deformation gradient tensor with $\mathbf{X}$ denoting the reference position. The corresponding Cauchy stress of the Neo-Hookean material is expressed as

$$
\boldsymbol{\sigma}(\mathbf{w})=J^{-1} \mathbf{P} \mathbf{F}^{T},
$$

where $J$ is the determinant of the deformation gradient tensor, $\mathbf{F}$, and $J=1$ for an incompressible Neo-Hookean material.. $\mathbf{P}=\frac{\partial W}{\partial \nabla_{\mathbf{X}} \mathbf{w}}$ is the first Piola-Kirchhoff stress.

The traction force on the particle-fluid interface is continuous, written as

$$
\boldsymbol{\sigma}(\mathbf{w}) \bullet \mathbf{n}_{p}=\boldsymbol{\sigma}_{f} \bullet \mathbf{n}_{f}+\boldsymbol{\sigma}_{E} \bullet \mathbf{n}_{f},
$$

where $\mathbf{n}_{p}$ is the unit normal vector directed from the particle surface into the fluid in the reference frame, $\boldsymbol{\sigma}_{f}=-p \mathbf{I}+\mu\left(\nabla \mathbf{u}+\nabla \mathbf{u}^{T}\right)$ and $\boldsymbol{\sigma}_{E}=\varepsilon_{f} \mathbf{E} \mathbf{E}-\frac{1}{2} \varepsilon_{f}(\mathbf{E} \bullet \mathbf{E}) \mathbf{I}$ are, respectively, the hydrodynamic stress tensor and the Maxwell stress tensor.

\section{Numerical Implementation and Code Validation}

The strongly coupled governing equations are solved based on the ALE method, in which the particle movement and deformation are tracked in a Lagrangian manner and the fluid flow and the electric field are simultaneously solved in an Eulerian framework. Detailed implementation of this numerical technique on the simulation of particle motion in viscous fluids was first introduced by $\mathrm{Hu}$ et al.[43-44] In the present study, a commercial finite-element package 
COMSOL (version 3.5a, www.comsol.com) operated with MATLAB (version 2009a, www.mathworks.com) in a high-performance cluster is exploited to simultaneously solve the particle-fluid-electric field coupled system using the ALE method. The computational domain consisting of the fluid domain $\Omega_{f}$ and the particle domain $\Omega_{p}$ (Figure 1) is discretized using quadratic triangular elements to satisfy the moving mesh requirement. A denser mesh is designated surrounding the particle to ensure that all the obtained results are fully converged and mesh-independent.

Without solving the solid phase, the developed ALE technique has been successfully employed to simulate the pressure-driven motion of rigid particles in a cylindrical channel [47] and a converging-diverging microchannel [45], which indicate, respectively, good agreements with analytical solutions and experimental data. In addition, the electrokinetic motions of rigid particles in microchannels [37-41] and nanopores [48] have been comprehensively studied using the ALE technique, which reveals that the DEP effect must be taken into account in the modeling of electrokinetic motion of rigid particles in microfluidic devices.

In order to validate the present numerical model for deformable particles, we previously reproduced the deformation of a RBC under the stress generated by optical tweezers [49]. The prediction was in good agreement with the experimental date of Mills et al. [50]. In addition, we simulate the deformation of a circular particle subjected to a shear-driven flow, which is compared to Gao and Hu's [32] numerical results obtained by their own ALE code. It is found that the circular particle is deformed to a perfect ellipse when the inertial force is negligible under very low Reynolds number. Figure 2 shows the stretch ratio of a circular particle as a function of the capillary number, which indicates a good agreement between our numerical results and Gao and Hu's results [32]. Here, the stretch ratio is defined as $D=\left(D_{1}-D_{2}\right) /\left(D_{1}+D_{2}\right)$, where $D_{1}$ and $D_{2}$ are, respectively, the lengths of the major axis and the minor axis of the deformed ellipse. The capillary number is defined as $C a=\mu \gamma / G_{0}$, where $\gamma$ is the shear rate of the flow. We further verify the validity of the present numerical model to simulate the electrokinetic motion of a deformable particle in a microchannel. Here, we consider a circular particle translating along the centerline of a slit channel and assume the shear modulus of the particle is very large $\left(G_{0}=337 \mathrm{~Pa}\right)$ which refers to a rigid particle. Figure 3 shows the dimensionless particle velocity normalized by $\varepsilon_{f} \zeta_{p} E / \mu$ as a function of the ratio of the particle diameter to the height of the microchannel. It is shown that the numerical results obtained by the present numerical model for deformable particles also recover the predictions obtained by previous numerical models for the limiting case of rigid particles [37-41].

\section{Results and Discussion}

The physical properties of the aqueous solution used in the present study include the fluid density, $\rho_{f}=\rho_{p}=1 \times 10^{3} \mathrm{~kg} / \mathrm{m}^{3}$, the fluid viscosity, $\mu=1 \times 10^{-3} \mathrm{~Pa} \cdot \mathrm{s}$ and the fluid permittivity, $\varepsilon_{f}=$ $7.08 \times 10^{-10} \mathrm{~F} / \mathrm{m}$. The particle of radius $a=1 \mu \mathrm{m}$ and length $L_{p}=12 \mu \mathrm{m}$ is initially positioned at $\left(x_{p 0}, y_{p 0}\right)=(-20 \mu \mathrm{m}, 0)$. The length of the slit channel is $L=100 \mu \mathrm{m}$ and the channel is assumed to be uncharged without further specification. The particle is always moving from left to right in the present study. In the following, the importance of the DEP effect is first demonstrated. Subsequently, the effects due to the shear modulus and the zeta potential of the particle, the applied electric field, and the solid boundary on the particle deformation are investigated.

\subsection{The Effect of DEP}


We assume the initial particle orientation with respect to the centerline of the microchannel is $\theta_{p 0}=60^{\circ}$. The externally applied electric field, the zeta potential of the particle, and the height of the microchannel are, respectively, $E=10 \mathrm{KV} / \mathrm{m}, \zeta_{p}=-50 \mathrm{mV}$ and $d=50 \mu \mathrm{m}$. Figure 4 shows the dynamics of a hyperelastic particle when $G_{0}=25 \mathrm{~Pa}$ (Figures $4 \mathrm{a}$ and $4 \mathrm{~d}$ ), $G_{0}=50 \mathrm{~Pa}$ (Figures $4 \mathrm{~b}$ and $4 \mathrm{e}$ ) and $G_{0}=500 \mathrm{~Pa}$ (Figures $4 \mathrm{c}$ and $4 \mathrm{f}$ ) at seven different time steps (from left to right): $t$ $=0,12,24,36,48,72$ and $100 \mathrm{~ms}$. The left (a, b, and c) and right (d, e, and f) columns in Figure 4 are the results obtained by the model with and without considering the DEP effect. When the DEP effect is not considered, the second term, $\boldsymbol{\sigma}_{E} \mathbf{n}_{f}$, on the right-hand-side of equation (12) is dropped. For the low shear modulus of $G_{0}=25 \mathrm{~Pa}$ (Figures $4 \mathrm{a}$ and $4 \mathrm{~d}$ ), the particle deforms significantly at the beginning $(t=12 \mathrm{~ms})$. In the absence of the DEP effect (Figure 4d), the particle remains curved while translating. However, with the DEP effect (Figure 4a), the particle rotates clockwise due to a net torque, causing an alignment to the external electric field. This DEP particle alignment phenomenon has been experimentally observed in our previous study and also predicted using the numerical model for rigid cylindrical particles [37]. In addition, van den Heuvel et al. [35] also experimentally observed the DEP alignment of rod-like microtubules. Interestingly, the particle deformation decreases during the alignment process, which is attributed to the decrease in the non-uniformity of the electric field on the particle surface. As the shear modulus increases, the particle becomes more rigid, which in turn decreases the deformation, as shown in Figure $4 \mathrm{~b}$. Figure $4 \mathrm{c}$ demonstrates that the particle with $G_{0}=500 \mathrm{~Pa}$ can be regarded as a rigid particle. However, the DEP particle alignments predicted in Figures 4a, $4 \mathrm{~b}$ and $4 \mathrm{c}$ almost follow the same tendency. When the term $\boldsymbol{\sigma}_{E}\left[\mathbf{n}_{f}\right.$ is removed from the righthand-side of equation (12) to neglect the DEP effect, the particle alignment to the applied electric field by rotation is not reflected in Figures $4 \mathrm{~d}$, $4 \mathrm{e}$ or $4 \mathrm{f}$. Thus, the DEP effect is responsible for the DEP particle alignment by rotation and consequent relaxation of particle deformation.

If the initial particle orientation with respect to the centerline of the microchannel is $\theta_{p 0}=90^{\circ}$, the torque acting on the particle is zero because of a symmetric distribution of the DEP force on the particle surface with respect to the centerline of the microchannel. As a result, the particle cannot be aligned to the external electric field at this singular initial orientation in the absence of any perturbation. Figure 5a shows the equilibrium particle shape when $E=20 \mathrm{KV} / \mathrm{m}, G_{0}=25$ $\mathrm{Pa}, \zeta_{p}=-50 \mathrm{mV}$ and $d=50 \mu \mathrm{m}$. When the particle moves from left to right, its equilibrium deformation is in a "C" shape, which is symmetric with respect to the centerline of the microchannel. This prediction is very similar to the numerical result obtained by Swaminathan $e t$ al. [36] without considering the DEP effect. However, Figure 5a also demonstrates that the particle deformation with considering the DEP effect is more significant than that without considering DEP effect. At the beginning, the distribution of the DEP force on the particle surface is symmetric with respect to the center of the undeformed particle, which does not contribute to the particle deformation. As the particle curls up due to a higher Smoluchowski slip velocity at the two ends of the particle, the electric field around the particle becomes asymmetric with respect to the center of the deformed particle. Accordingly, the distribution of the DEP force on the particle surface becomes asymmetric with respect to the center of the deformed particle, as shown in Figure 5b. This DEP effect further aids the particle deformation. Therefore, the particle deformation is mainly due to the inherently non-uniform Smoluchowski slip velocity on the particle surface and also the induced asymmetric DEP force with respect to the center of the deformed particle. 
Figure 6 shows the effect of the applied electric field on the steady averaged particle velocity in the $x$ direction normalized by $U_{0}=\varepsilon_{f} \zeta_{p} E / \mu$ when $G_{0}=25 \mathrm{~Pa}, \theta_{p 0}=90^{\circ}, \zeta_{p}=-50 \mathrm{mV}$ and $d$ $=50 \mu \mathrm{m}$. When the particle reaches its equilibrium state, its steady averaged particle velocity in the $x$ direction is defined as

$$
U_{p}=\frac{\int \frac{\partial w_{x}}{\partial t} d \Omega_{p}}{\int 1 d \Omega_{p}},
$$

where $w_{x}$ is the particle displacement in the $x$ direction. The DEP effect is relatively weak when the applied electric field is relatively low. Hence, the averaged particle velocity with considering the DEP is very close to that without considering the DEP when the applied electric field is $E=5$ $\mathrm{KV} / \mathrm{m}$. However, the DEP effect is proportional to the square of the electric field strength. As the electric field increases, the averaged particle velocity with considering the DEP significantly deviates from that without considering the DEP. As the net DEP force is directed toward the negative $x$ direction, the DEP effect tends to slow down the particle motion, as shown in Figure 6 . The DEP effect plays an important role in the particle deformation and the particle mobility. As a result, the DEP effect must be taken into account in the modeling of electrokinetic motion of deformable particles in microfluidics, and is thus considered in all the following simulations.

\subsection{The Effect of the Shear Modulus of the Particle}

As indicated in Figure 4, the particle deformation increases as the shear modulus of the particle decreases. However, the particle deformation decreases owing to the particle alignment to the electric field. To assess the influence of DEP force on particle deformation without particle rotation and alignment, we set the initial particle orientation to $\theta_{p 0}=90^{\circ}$ and investigate the effect of the shear modulus of the particle on its deformation. Figure 7 shows the transient deformation of a hyperelastic particle when $E=10 \mathrm{KV} / \mathrm{m}, \zeta_{p}=-50 \mathrm{mV}, d=50 \mu \mathrm{m}, G_{0}=12.5 \mathrm{~Pa}$ (Figure 7a), $G_{0}=25 \mathrm{~Pa}$ (Figure 7b), $G_{0}=50 \mathrm{~Pa}$ (Figure 7c) and $G_{0}=100 \mathrm{~Pa}$ (Figure 7d). As the electric field near the two ends of the particle is higher than that in the other regions, the nonuniform Smoluchowski slip velocity on the particle surface thus generates a larger shear force at the two ends of the particle, which tends to curl up the particle to a "C" shape as it moves from left to right. As mentioned earlier in Figure 5, the DEP effect also enhances the particle deformation. Obviously, a smaller shear modulus implies a softer particle, which is easier to deform, as shown in Figure 7.

\subsection{The Effect of the Applied Electric Field}

The externally applied electric field can effectively change the Smoluchowski slip velocity on the particle surface and also the DEP force acting on the particle, which in turn affects the particle deformation. Figure 8 shows the transient deformation of a hyperelastic particle under two different electric fields $E=20 \mathrm{KV} / \mathrm{m}$ (Figure 8a) and $E=30 \mathrm{KV} / \mathrm{m}$ (Figure 8b) when $G_{0}=$ $25 \mathrm{~Pa}, \theta_{p 0}=90^{\circ}, \zeta_{p}=-50 \mathrm{mV}$ and $d=50 \mu \mathrm{m}$. Clearly, the equilibrium particle shape when $E=$ $10 \mathrm{KV} / \mathrm{m}$ (Figure $7 \mathrm{~b}$ ) is less deformed than the case of $E=20 \mathrm{KV} / \mathrm{m}$ (Figure 8a), which is less deformed than the case of $E=30 \mathrm{KV} / \mathrm{m}$ (Figure 8b). Therefore, control of the external electric field is a very efficient way to manipulate the particle deformation.

Fluid-structure interaction is a two-way coupling problem. Hence, the particle deformation also significantly affects the flow field around the particle. Figure 9 illustrates the evolution of 
the flow field around the particle when $E=20 \mathrm{KV} / \mathrm{m}, G_{0}=25 \mathrm{~Pa}, \theta_{p 0}=90^{\circ}, \zeta_{p}=-50 \mathrm{mV}$ and $d$ $=50 \mu \mathrm{m}$. Under the joint effects of the particle movement and the EOF in the vicinity of the charged particle, a vortex is induced at either end of the particle as shown in Figure 9a. As the particle curls up, the two ends of the particle approach each other, which in turn brings the two vortices closer to each other as depicted in Figures 9b, 9c and 9d. The fluid outside the induced vortex moves very slowly in the direction opposite to the particle movement. This is attributed to the EOF induced by the surface charge of the particle.

We further investigate the effect of the electric field on the particle behavior when the particle is not initially placed perpendicular to the centerline of the microchannel. In such case, the particle alignment to the applied electric field and possible relaxation of particle deformation come into play. Figure 10 shows the transient behavior of a hyperelastic particle under two different electric fields $E=20 \mathrm{KV} / \mathrm{m}$ (Figure 10a) and $E=30 \mathrm{KV} / \mathrm{m}$ (Figure 10b) when $G_{0}=50$ $\mathrm{Pa}, \theta_{p 0}=60^{\circ}, \zeta_{p}=-50 \mathrm{mV}$ and $d=50 \mu \mathrm{m}$. Note that the same case under $E=10 \mathrm{KV} / \mathrm{m}$ is shown in Figure 4b. A higher electric field leads to a higher Smoluchowski slip velocity at the two ends of the particle, which accordingly induces a larger particle deformation at the beginning. Subsequently, the particle is aligned to the electric field due to the DEP effect which increases with the electric field. Figure 10 demonstrates that a higher electric field leads to a faster particle alignment, which has also been observed in our previous experimental study [37]. When the particle becomes nearly parallel to the applied electric field, the particle deformation is almost eliminated.

\subsection{The Effect of the Zeta Potential of the Particle}

Figure 11 shows the effect of the zeta potential of the particle on the particle deformation when $E=20 \mathrm{KV} / \mathrm{m}, G_{0}=25 \mathrm{~Pa}, \theta_{p 0}=90^{\circ}$ and $d=50 \mu \mathrm{m}$. In order to compare the equilibrium particle shapes at approximately the same location under different zeta potentials, the time step is accordingly scaled based on the magnitude of the zeta potential of the particle. It is shown in equation (8) that the Smoluchowski slip velocity is proportional to the applied electric field and also the zeta potential of the particle. Therefore, a higher zeta potential leads to a larger nonuniformity of the Smoluchowski slip velocity on the particle surface, which in turn increases the particle deformation. It should be noticed that the electrokinetics-induced particle deformation is totally different from that subjected to a pressure-driven flow. According to the experimental observations [23-24], the present cylindrical particle subjected to a pressure-driven flow is expected to curl up as a horizontally reversed " $C$ " shape when it moves from left to right.

\subsection{The Effect of the Rigid Channel Boundary}

Figure 12 shows the boundary effect on the particle deformation when $E=10 \mathrm{KV} / \mathrm{m}, G_{0}=25$ $\mathrm{Pa}, \theta_{p 0}=90^{\circ}, \zeta_{p}=-50 \mathrm{mV}$ and $t=50 \mathrm{~ms}$. When the distance between the rigid walls decreases, the electric field between the particle and the rigid wall is enhanced, which in turn increases the Smoluchowski slip velocity on the particle surface. As a result, the particle deformation slightly increases when the boundary effect becomes more pronounced. However, the particle velocity decreases mainly due to the increasing wall retardation effect arising from the stationary rigid boundary.

In all of the above results, the zeta potential of the rigid wall is $\zeta_{w}=0$. In order to clearly assess the effects of charged walls, we consider a case when the zeta potential of the particle and the rigid wall are, respectively, is $\zeta_{p}=0$ and $\zeta_{w}=50 \mathrm{mV}$, and keep all the other conditions in Figure 12 unchanged. In this case, the particle motion is driven by the EOF originating from the 
channel walls. As the velocity profile of EOF is plug-like, the flow field around the particle suspended in an unbounded domain is uniform, which is unable to induce particle deformation. It is indeed found that the particle does not deform when $d=50 \mu \mathrm{m}$ and $40 \mu \mathrm{m}$, where the boundary effect is very small. As the distance between the two rigid walls further decreases to $d$ $=30 \mu \mathrm{m}$ and $20 \mu \mathrm{m}$, the confinement effect increases and the particle starts to deform. However, the particle deformation is still much smaller than those shown in Figure 12. Thus, the effect of EOF in a charged confined channel on particle deformation can be largely neglected in comparison to the effects of particle electrophoresis.

\section{Concluding Remarks}

We have developed a numerical model to study the electrokinetic motion of a $2 \mathrm{D}$ hyperelastic particle, focusing on the electrokinetics-induced particle deformation in a confined microchannel. The inertial force is negligible due to a very low Reynolds number in microfluidics. The shear force arising from the non-uniform Smoluchowski slip velocity on the particle surface is responsible for the electrokinetics-induced particle deformation. Furthermore, the asymmetric DEP force with respect to the center of the deformed particle also enhances the particle deformation when symmetries of the particle prevent its rotation.

When the cylindrical particle is initially perpendicular to the applied electric field, it curls up like a "C" shape as it moves from left to right. The electrokinetics-induced particle deformation is opposite to the particle deformation subjected to a pressure-driven flow when the particle moves in the same direction. A particle with a smaller shear modulus is softer, which is easier to deform. Also, the applied electric field, the zeta potential of the particle and the boundary effect play an important role in the particle deformation. However, the plug-like EOF arising from the charged channel walls indicates a minor effect on the particle deformation. When the cylindrical particle is not initially perpendicular to the applied electric field, the DEP effect progressively aligns the particle with its longest axis parallel to the applied electric field. Furthermore, a higher electric field leads to a faster particle alignment, which is in good agreement with our previous experimental study [37]. Interestingly, the particle alignment decreases the particle deformation because of a higher uniformity of the electric field. The developed numerical model may prove useful in understanding and controlling the electrokinetics-induced deformation of biological cells, for example RBC, in microfluidics.

\section{Acknowledgment}

We acknowledge Prof. Howard H. Hu at University of Pennsylvania for discussion. This work is supported by the World Class University Grant No. R32-2008-000-20082-0 of the Ministry of Education, Science and Technology of Korea. 


\section{References}

[1] Dolnik, V., Liu, S. R., Jovanovich, S., Electrophoresis 2000, 21, 41-54.

[2] Leopold, K., Dieter, B., Ernst, K., Electrophoresis 2004, 25, 2282-2291.

[3] Kang, Y. J., Li, D. Q., Microfluid. Nanofluid. 2009, 6, 431-460.

[4] Keh, H. J., Anderson, J. L., J. Fluid Mech. 1985, 153, 417-439.

[5] Ye, C. Z., Li, D. Q., J. Colloid Interface Sci. 2004, 272, 480-488.

[6] Ye, C. Z., Xuan, X. C., Li, D. Q., Microfluid. Nanofluid. 2005, 1, 234-241.

[7] Unni, H. N., Keh, H. J., Yang, C., Electrophoresis 2007, 28, 658-664.

[8] Hsu, J. P., Kuo, C. C., J. Phys. Chem. B 2006, 110, 17607-17615.

[9] Hsu, J. P., Yeh, L. H., Ku, M. H., Colloid Polym. Sci. 2006, 284, 886-892.

[10] Yariv, E., Phys. Fluids 2006, 18, 031702.

[11] Davison, S. M., Sharp, K. V., Microfluidics and Nanofluidics 2008, 4, 409-418.

[12] Ennis, J., Anderson, J. L., J. Colloid Interface Sci. 1997, 185, 497-514.

[13] Xuan, X. C., Raghibizadeh, R., Li, D., J. Colloid Interface Sci. 2006, 296, 743-748.

[14] Zhu, J., Xuan, X., J. Colloid Interface Sci. 2009, 340, 285-290.

[15] Liang, L. T., Ai, Y., Zhu, J. J., Qian, S., Xuan, X. C., J. Colloid Interface Sci. 2010, 347, 142-146.

[16] Gallo-Villanueva, R. C., Rodriguez-Lopez, C. E., Diaz-De-La-Garza, R. I., Reyes-Betanzo, C., Lapizco-Encinas, B. H., Electrophoresis 2009, 30, 4195-4205.

[17] Martinez-Lopez, J. I., Moncada-Hernandez, H., Baylon-Cardiel, J. L., Martinez-Chapa, S. O., Rito-Palomares, M., Lapizco-Encinas, B. H., Anal. Bioanal. Chem. 2009, 394, 293-302.

[18] Gloria, O., Christina, S., Matthew, B. K., Anubhav, T., Anuj, C., Electrophoresis 2008, 29, 1152-1163.

[19] Kang, K. H., Kang, Y. J., Xuan, X. C., Li, D. Q., Electrophoresis 2006, 27, 694-702.

[20] Thwar, P. K., Linderman, J. J., Burns, M. A., Electrophoresis 2007, 28, 4572-4581.

[21] Risso, F., Colle-Paillot, F., Zagzoule, M., J. Fluid Mech. 2006, 547, 149-173.

[22] Dondorp, A. M., Angus, B. J., Chotivanich, K., Silamut, K., Ruangveerayuth, R., Hardeman, M. R., Kager, P. A., Vreeken, J., White, N. J., Am. J. Trop. Med. Hyg. 1999, 60, 733737.

[23] Abkarian, M., Faivre, M., Stone, H. A., Proc. Natl. Acad. Sci. U. S. A. 2006, 103, 538-542. [24] Abkarian, M., Faivre, M., Horton, R., Smistrup, K., Best-Popescu, C. A., Stone, H. A., Biomed. Mater. 2008, 3, 034011.

[25] Korin, N., Bransky, A., Dinnar, U., J. Biomech. 2007, 40, 2088-2095.

[26] Tomaiuolo, G., Simeone, M., Martinelli, V., Rotoli, B., Guido, S., Soft Matter 2009, 5, 3736-3740.

[27] Tomaiuolo, G., Barra, M., Preziosi, V., Cassinese, A., Rotoli, B., Guido, S., Lab Chip 2011, DOI: 10.1039/C1030LC00348D.

[28] Chen, Y. C., Chen, G. Y., Lin, Y. C., Wang, G. J., Microfluid. Nanofluid. 2010, 9, 585-591.

[29] Eggleton, C. D., Popel, A. S., Phys. Fluids 1998, 10, 1834-1845.

[30] Secomb, T. W., Styp-Rekowska, B., Pries, A. R., Ann. Biomed. Eng. 2007, 35, 755-765.

[31] Doddi, S. K., Bagchi, P., Phys. Rev. E 2009, 79, 046318.

[32] Gao, T., Hu, H. H., J. Comput. Phys. 2009, 228, 2132-2151.

[33] MacMeccan, R. M., Clausen, J. R., Neitzel, G. P., Aidun, C. K., J. Fluid Mech. 2009, 618, 13-39. 
[34] Sugiyama, K., Ii, S., Takeuchi, S., Takagi, S., Matsumoto, Y., J. Comput. Phys. 2011, 230, 596-627.

[35] van den Heuvel, M. G. L., Bondesan, R., Lagomarsino, M. C., Dekker, C., Phys. Rev. Lett. $2008,101,118301$.

[36] Swaminathan, T. N., Gao, T., Hu, H. H., J. Colloid Interface Sci. 2010, 346, 270-276.

[37] Ai, Y., Beskok, A., Gauthier, D. T., Joo, S. W., Qian, S., Biomicrofluidics 2009, 3, 044110.

[38] Ai, Y., Joo, S. W., Jiang, Y., Xuan, X., Qian, S., Electrophoresis 2009, 30, 2499-2506.

[39] Ai, Y., Park, S., Zhu, J., Xuan, X., Beskok, A., Qian, S., Langmuir 2010, 26, 2937-2944.

[40] Ai, Y., Qian, S., J. Colloid Interface Sci. 2010, 346, 448-454.

[41] Ai, Y., Qian, S., Liu, S., Joo, S. W., Biomicrofluidics 2010, 4, 013201.

[42] Ai, Y., Liu, J., Zhang, B., Qian, S., Anal. Chem. 2010, 82, 8217-8225.

[43] Hu, H. H., Joseph, D. D., Crochet, M. J., Theor. Comput. Fluid Dyn. 1992, 3, 285-306.

[44] Hu, H. H., Patankar, N. A., Zhu, M. Y., J. Comput. Phys. 2001, 169, 427-462.

[45] Ai, Y., Joo, S. W., Jiang, Y., Xuan, X., Qian, S., Biomicrofluidics 2009, 3, 022404.

[46] Dao, M., Lim, C. T., Suresh, S., Journal of the Mechanics and Physics of Solids 2003, 51, 2259-2280.

[47] Quddus, A. N., Moussa, W. A., Bhattacharjee, S., J. Colloid Interface Sci. 2008, 317, 620630.

[48] Ai, Y., Qian, S., Phys. Chem. Chem. Phys. 2011, DOI: 10.1039/c0cp02267e.

[49] Mauroy, B., ESAIM: Proc. 2008, 23, 48-65.

[50] Mills, J. P., Qie, L., Dao, M., Lim, C. T., Suresh, S., MCB 2004, 1, 169-180.

[51] Liu, Qian and Bau, 2007, Biophysical Journal, 1164-1177. 


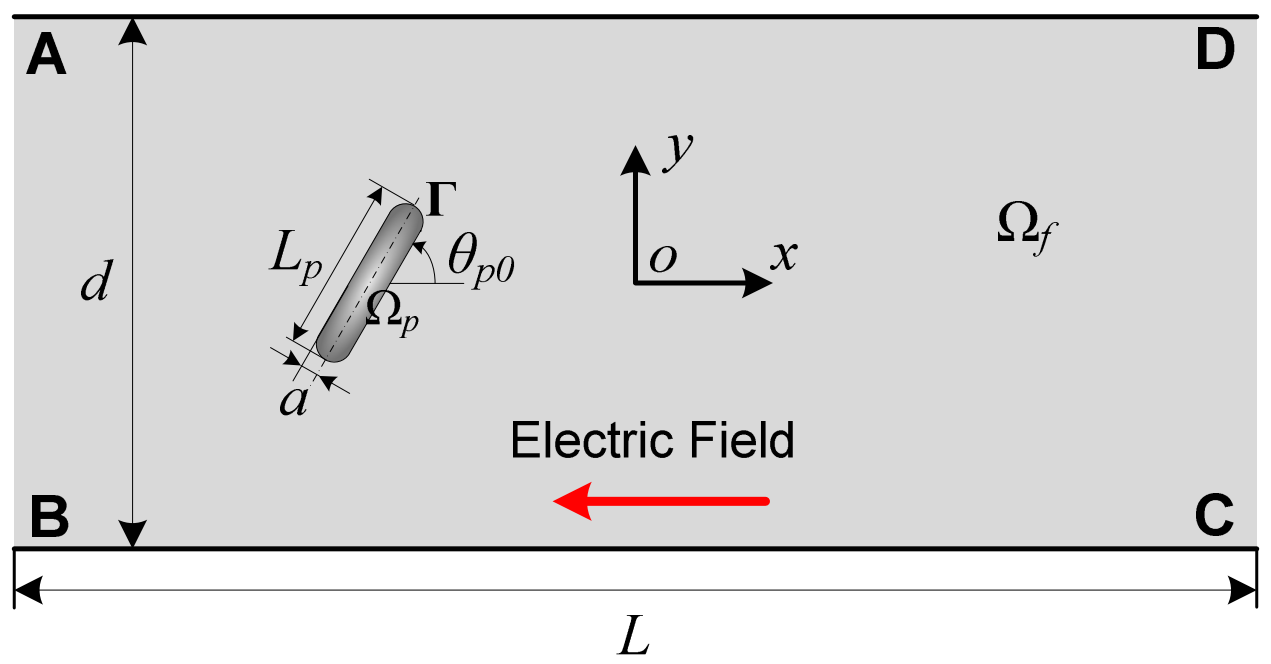

Figure 1 Schematics of the electrokinetic motion of a deformable cylindrical particle in a straight microchannel. 


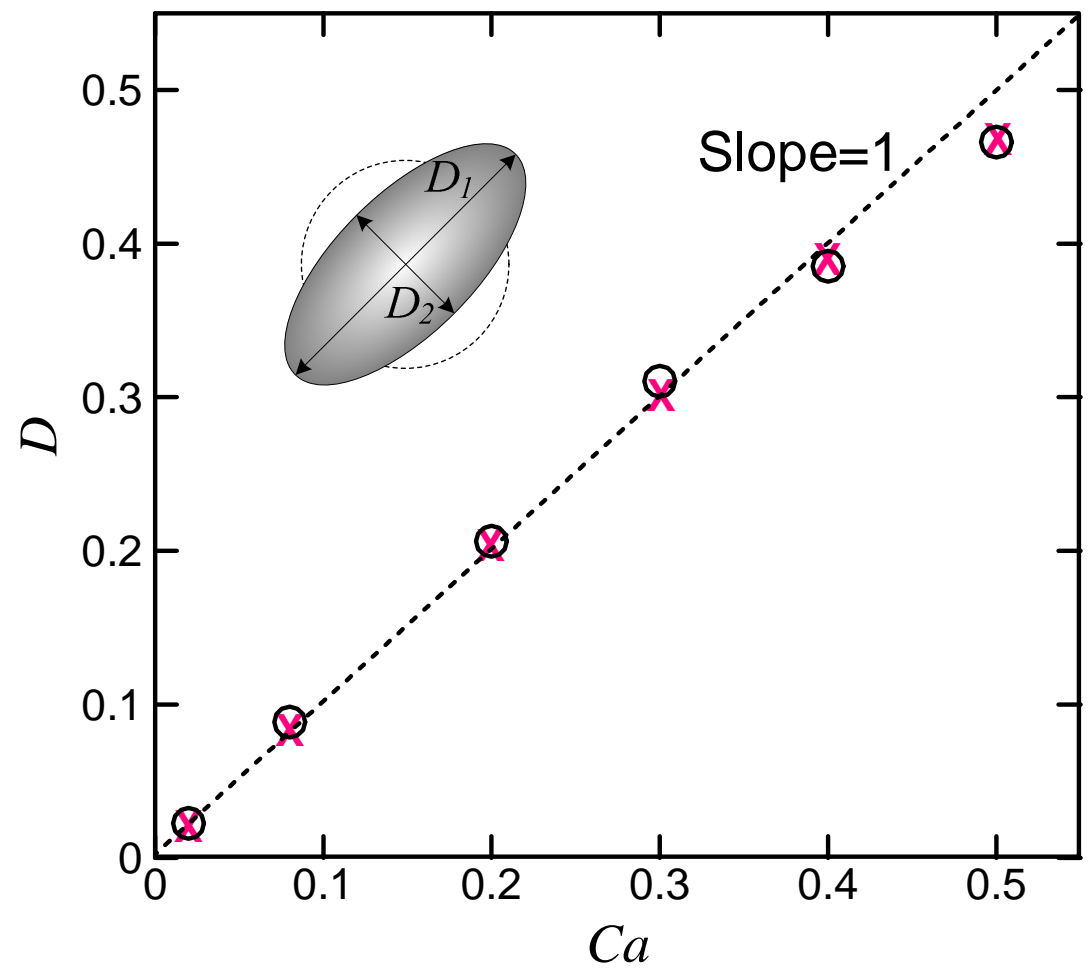

Figure 2 Stretch ratio of a circular particle subjected to a shear-driven flow as a function of capillary number. Circles and crosses respresent, respectively, our numerical results and the results obtained by $\mathrm{Gao}$ and $\mathrm{Hu}$ [27]. The inset schematically illustrates the deformation of the circular particle into an ellipse. 


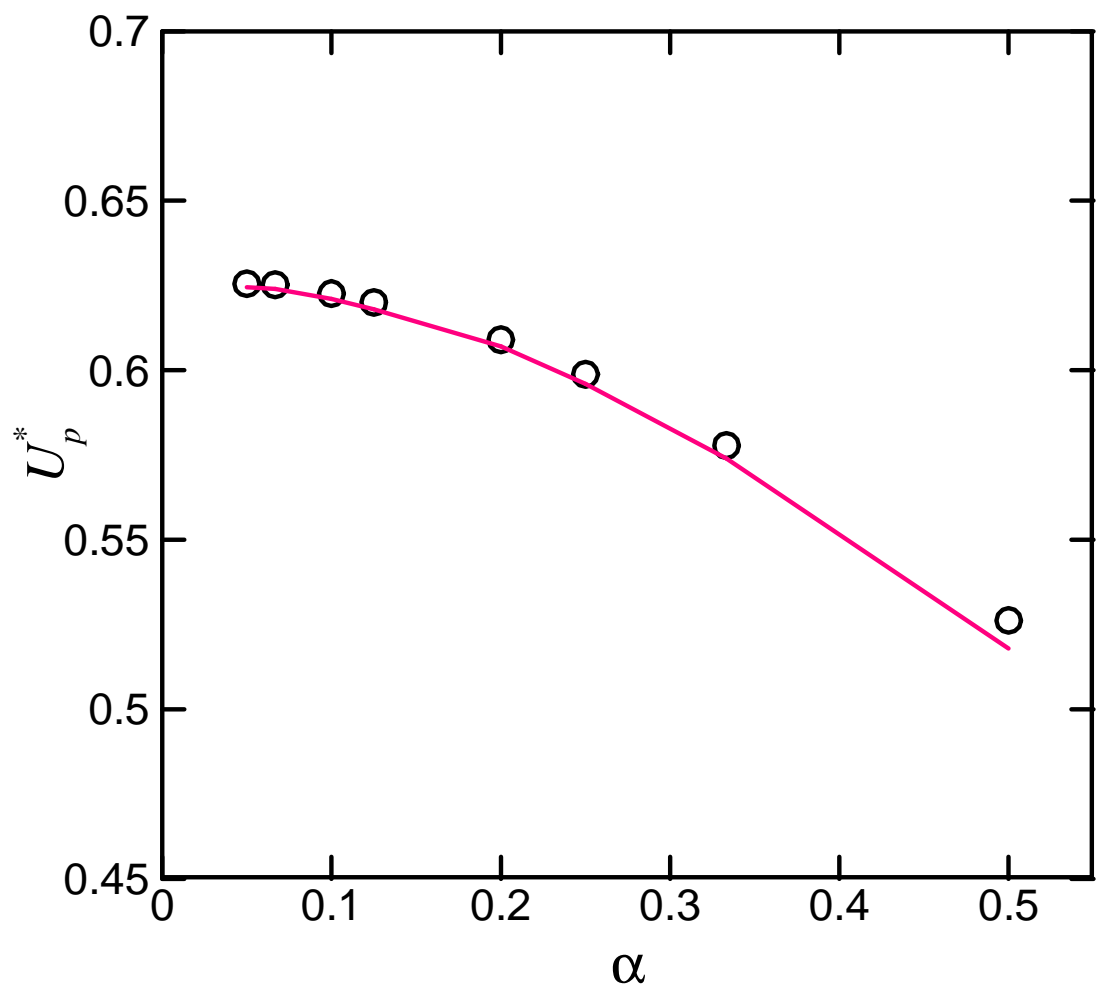

Figure 3 Dimensionless translational velocity normalized by $\varepsilon_{f} \zeta_{p} E / \mu$ of a circular particle translating along the centerline of a slit microchannel as a function of the ratio of the particle diameter to the height of the microchannel. Solid line and circles represent, respectively, the results obtained by the model for a rigid particle and the present model for a deformable particle with a very high shear modulus. The ratio of the zeta potential of the microchannel to that of the particle is 0.375 . 

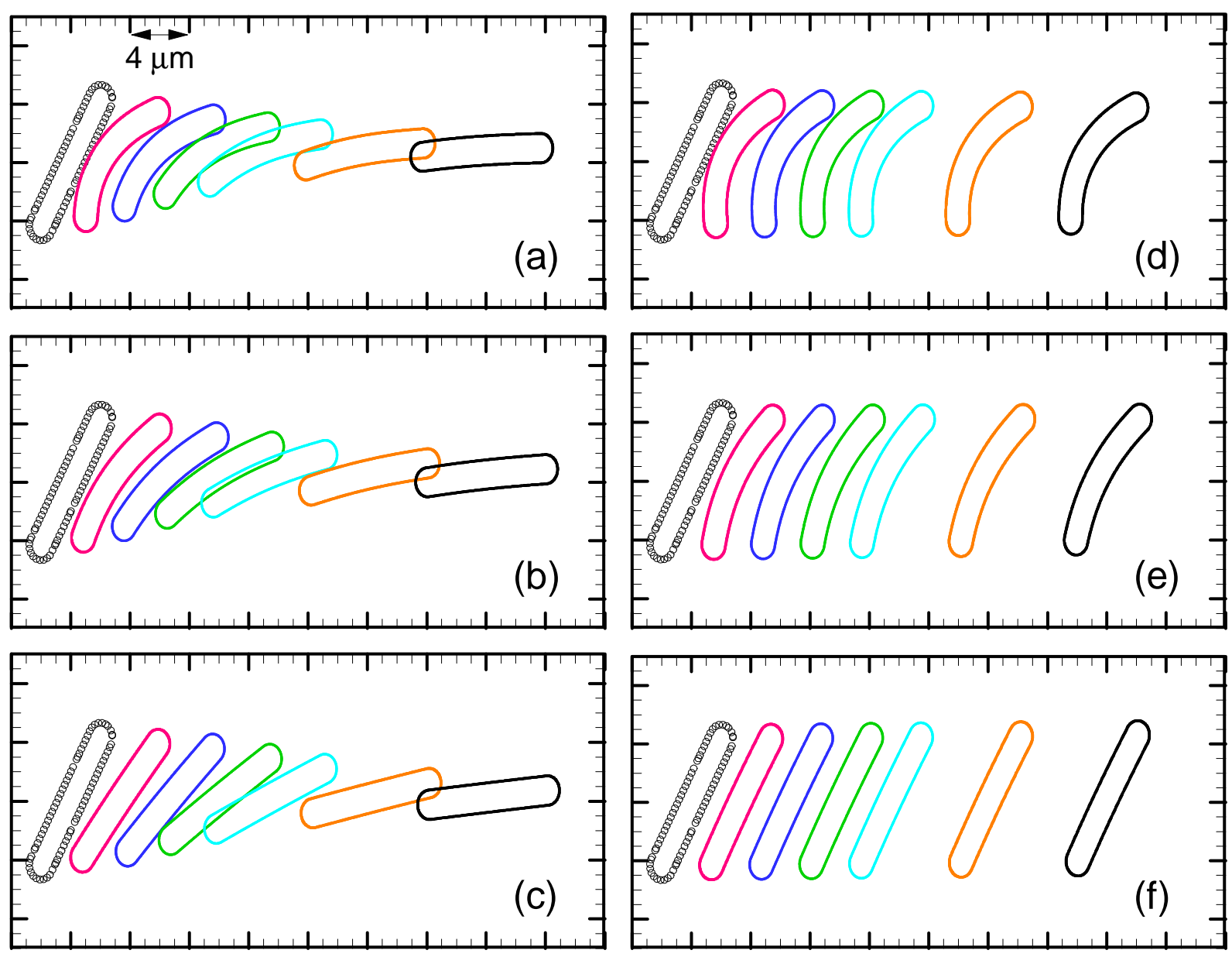

Figure 4 Dynamics of a hyperelastic particle when $G_{0}=25 \mathrm{~Pa}$ (a and d), $G_{0}=50 \mathrm{~Pa}$ (b and e) and $G_{0}=500 \mathrm{~Pa}$ (c and f) at seven different time steps (from left to right): $t=0,12,24,36,48,72$ and $100 \mathrm{~ms}$. The results in (a, b and c) and (d, e, and f) are, respectively, obtained by the models with and without considering the DEP effect. $E=10 \mathrm{KV} / \mathrm{m}, \theta_{p 0}=60^{\circ}, \zeta_{p}=-50 \mathrm{mV}$ and $d=50$ $\mu \mathrm{m}$. 

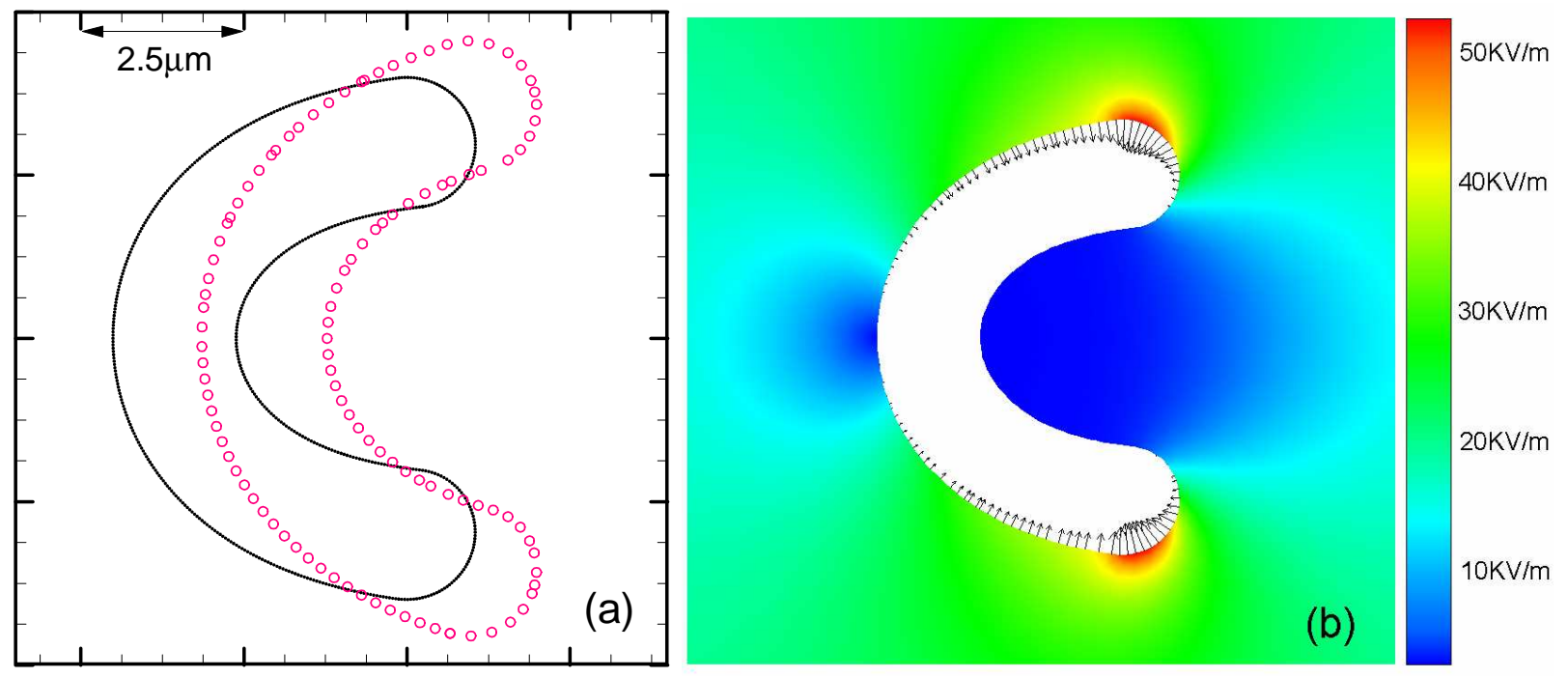

Figure 5 (a) Equilibrium shape of a hyperelastic particle when $E=20 \mathrm{KV} / \mathrm{m}, G_{0}=25 \mathrm{~Pa}, \theta_{p 0}=$ $90^{\circ}, \zeta_{p}=-50 \mathrm{mV}$ and $d=50 \mu \mathrm{m}$. Solid line and circles represent, respectively, the results obtained by the models with and without considering the DEP effect. (b) Distribution of the electric field around the deformed particle. The color levels denote the magnitude of the electric field strength and the arrows on the particle surface indicate the distribution of the DEP force. 


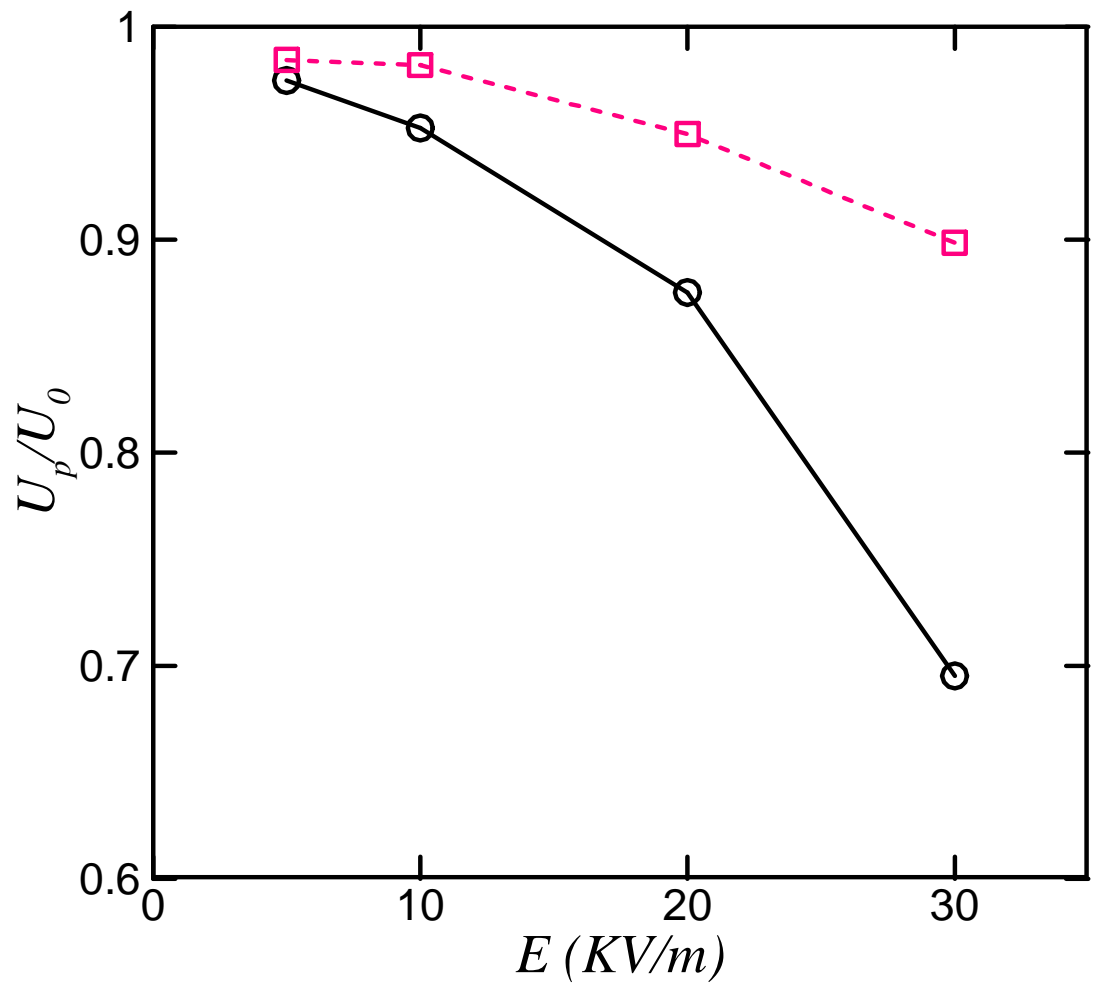

Figure 6 Steady averaged particle velocity in the $x$ direction normalized by $U_{0}=\varepsilon_{f} \zeta_{p} E / \mu$ as a function of the applied electric field when $G_{0}=25 \mathrm{~Pa}, \theta_{p 0}=90^{\circ}, \zeta_{p}=-50 \mathrm{mV}$ and $d=50 \mu \mathrm{m}$. Solid line with circles and dashed line with squares represent, respectively, the results obtained by the models with and without considering the DEP effect.
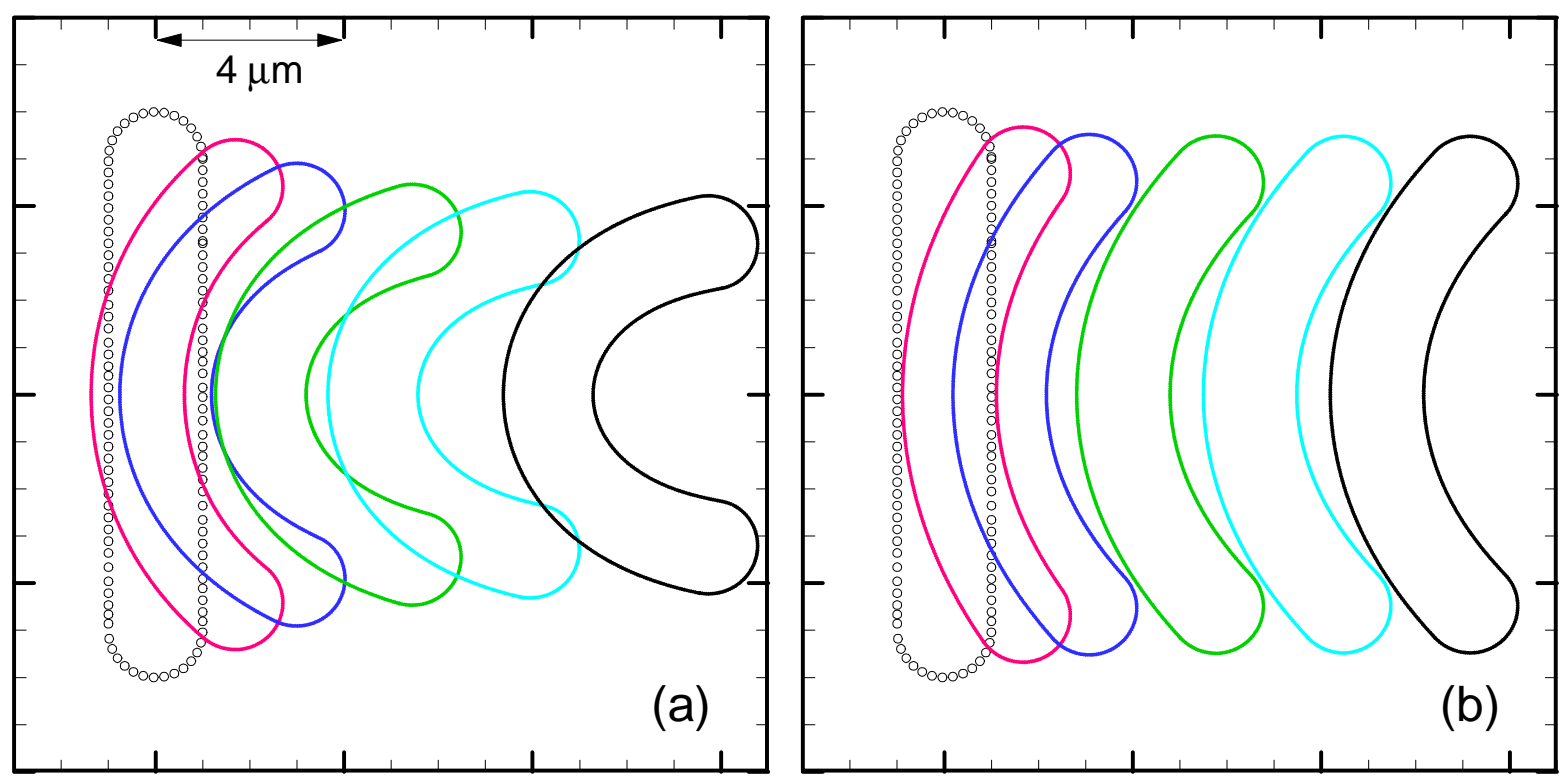

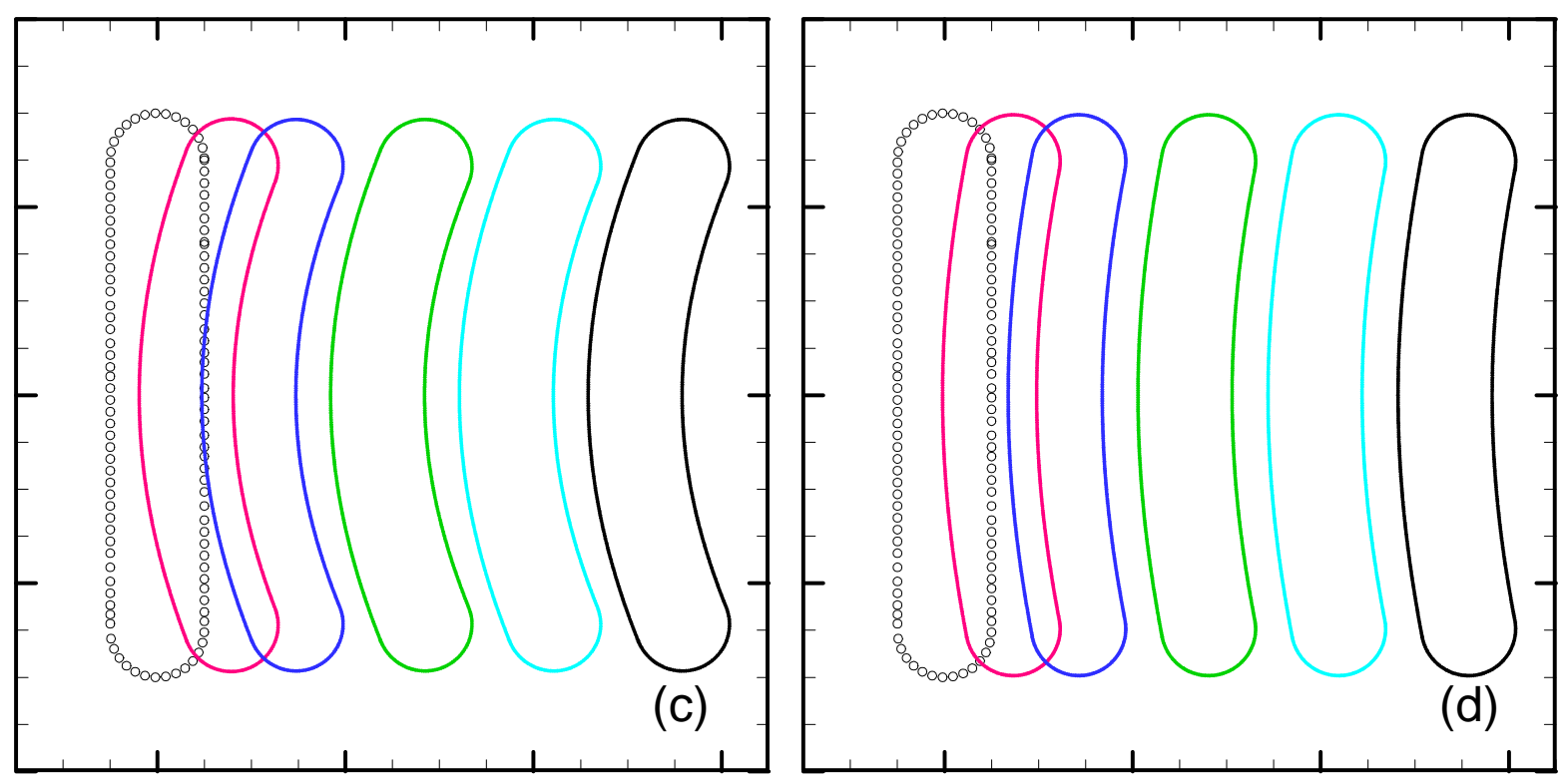

Figure 7 Deformation of a hyperelastic particle when $G_{0}=12.5 \mathrm{~Pa}(\mathrm{a}), G_{0}=25 \mathrm{~Pa}(\mathrm{~b}), G_{0}=50$ $\mathrm{Pa}(\mathrm{c})$ and $G_{0}=100 \mathrm{~Pa}(\mathrm{~d})$ at six different time steps (from left to right): $t=0,4,8,16,24$ and 32 ms. $E=10 \mathrm{KV} / \mathrm{m}, \theta_{p 0}=90^{\circ}, \zeta_{p}=-50 \mathrm{mV}$ and $d=50 \mu \mathrm{m}$. 

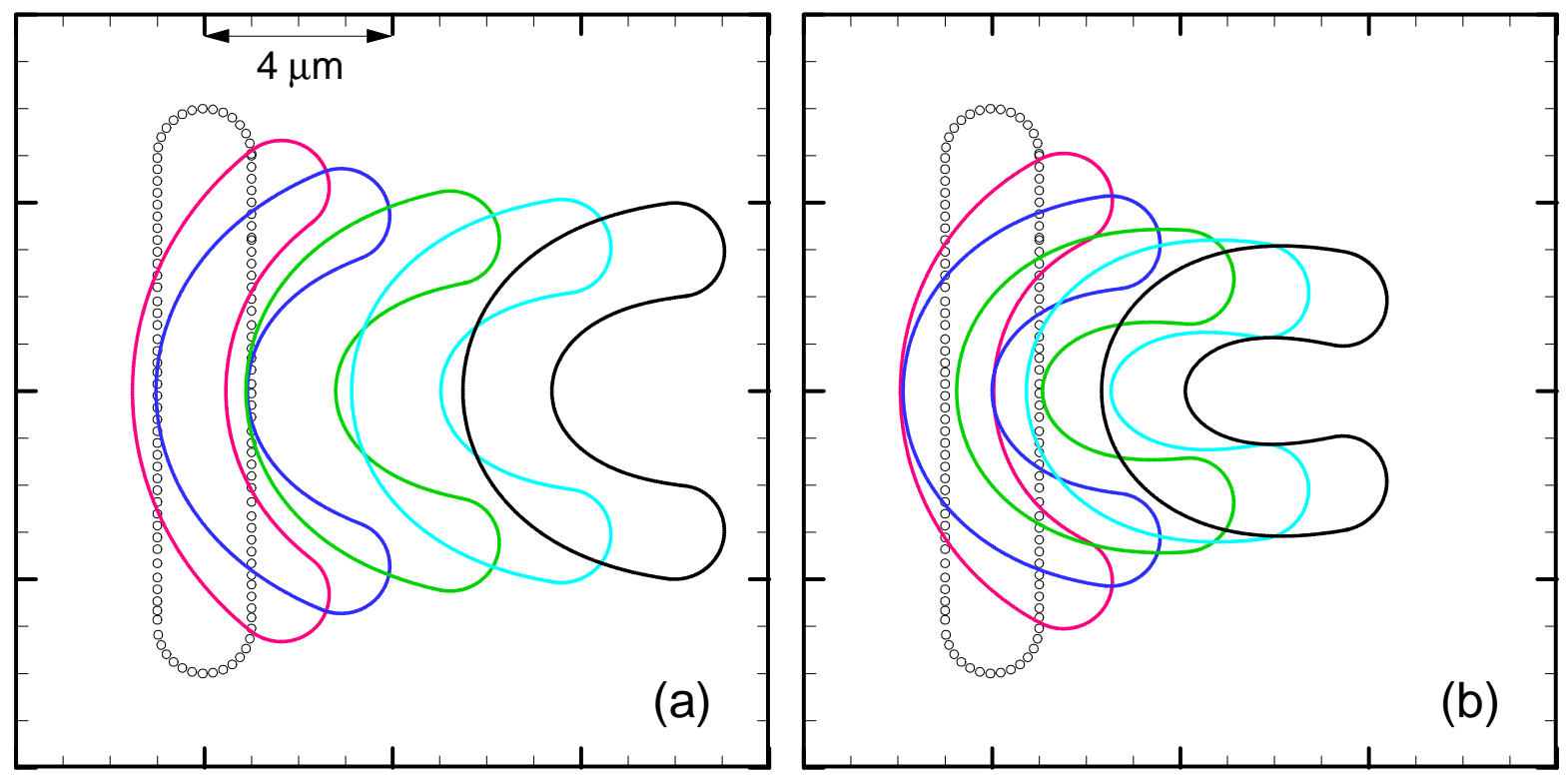

Figure 8 Deformation of a hyperelastic particle when $E=20 \mathrm{KV} / \mathrm{m}$ (a) and $E=30 \mathrm{KV} / \mathrm{m}$ (b) at six different time steps (from left to right): $t=0,2,4,8,12$ and $16 \mathrm{~ms}$ (a) and $t=0,1.33,2.67$, 5.33, 8 and $10.67 \mathrm{~ms} \mathrm{(b).} G_{0}=25 \mathrm{~Pa}, \theta_{p 0}=90^{\circ}, \zeta_{p}=-50 \mathrm{mV}$ and $d=50 \mu \mathrm{m}$. 


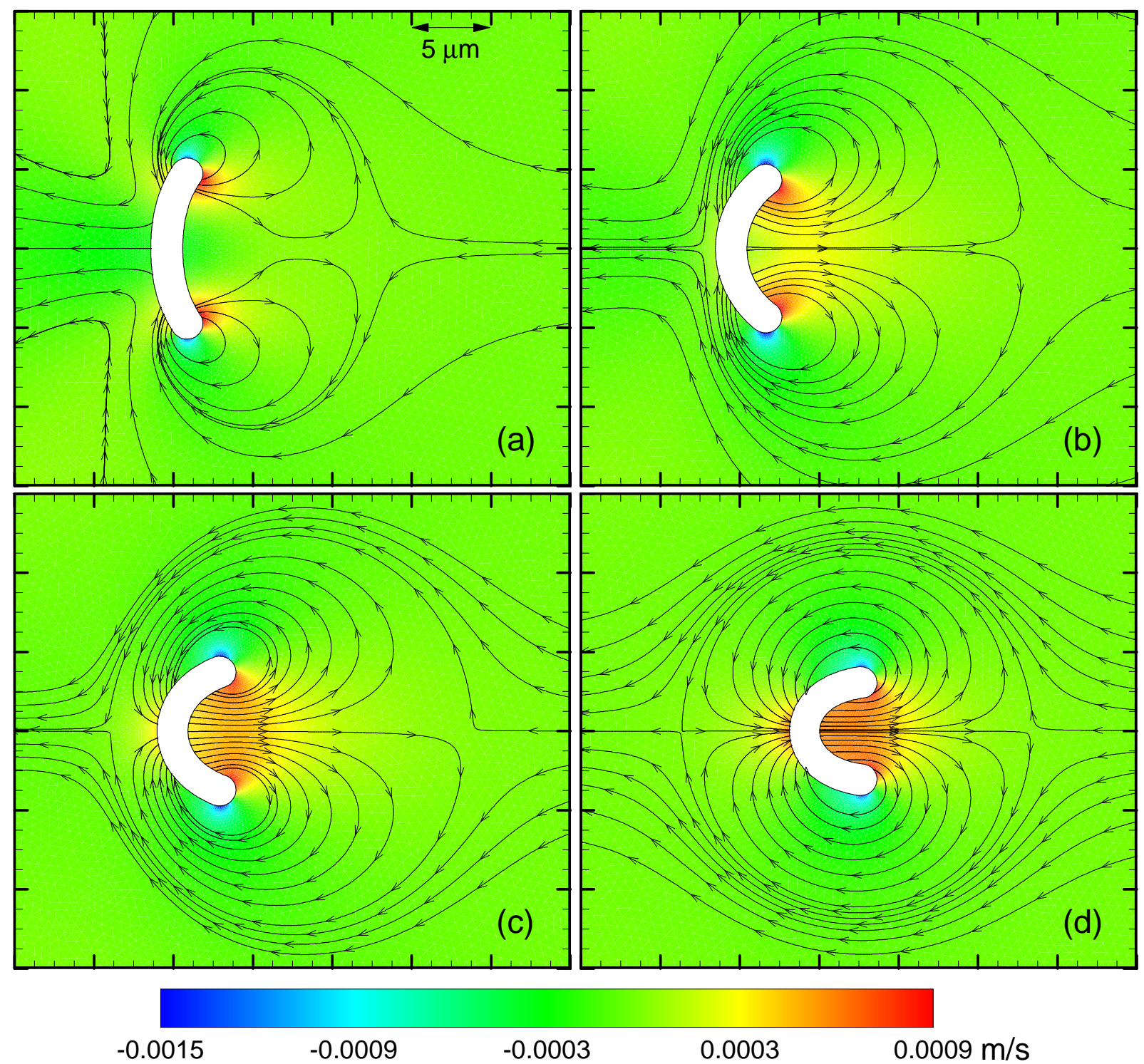

Figure 9 Flow field around a hyperelastic particle at $t=1 \mathrm{~ms}$ (a), $2 \mathrm{~ms}$ (b), $4 \mathrm{~ms}$ (c) and $12 \mathrm{~ms}$ (d) when $E=20 \mathrm{KV} / \mathrm{m}, G_{0}=25 \mathrm{~Pa}, \theta_{p 0}=90^{\circ}, \zeta_{p}=-50 \mathrm{mV}$ and $d=50 \mu \mathrm{m}$. The color levels denote the $x$-component flow velocity and the streamlines with arrows indicate the flow field. 

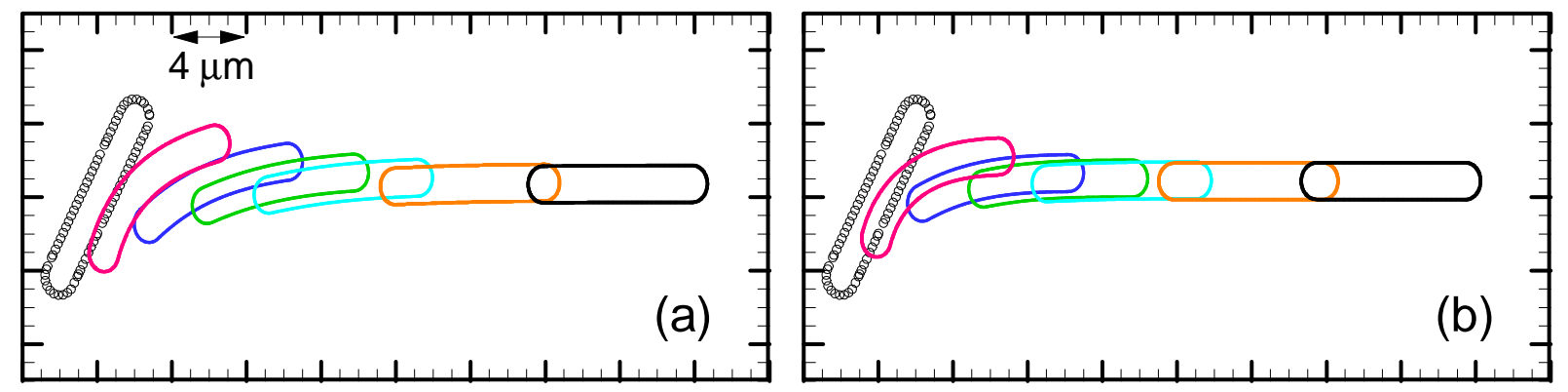

Figure 10 Dynamics of a hyperelastic particle when $E=20 \mathrm{KV} / \mathrm{m}$ (a) and $E=30 \mathrm{KV} / \mathrm{m}$ (b) at six different time steps (from left to right): $t=0,6,12,18,24,36$ and $50 \mathrm{~ms}$ (a) $t=0,4,8,12,16$, 24 and $33.3 \mathrm{~ms} . G_{0}=50 \mathrm{~Pa}, \theta_{p 0}=60^{\circ}, \zeta_{p}=-50 \mathrm{mV}$ and $d=50 \mu \mathrm{m}$. 


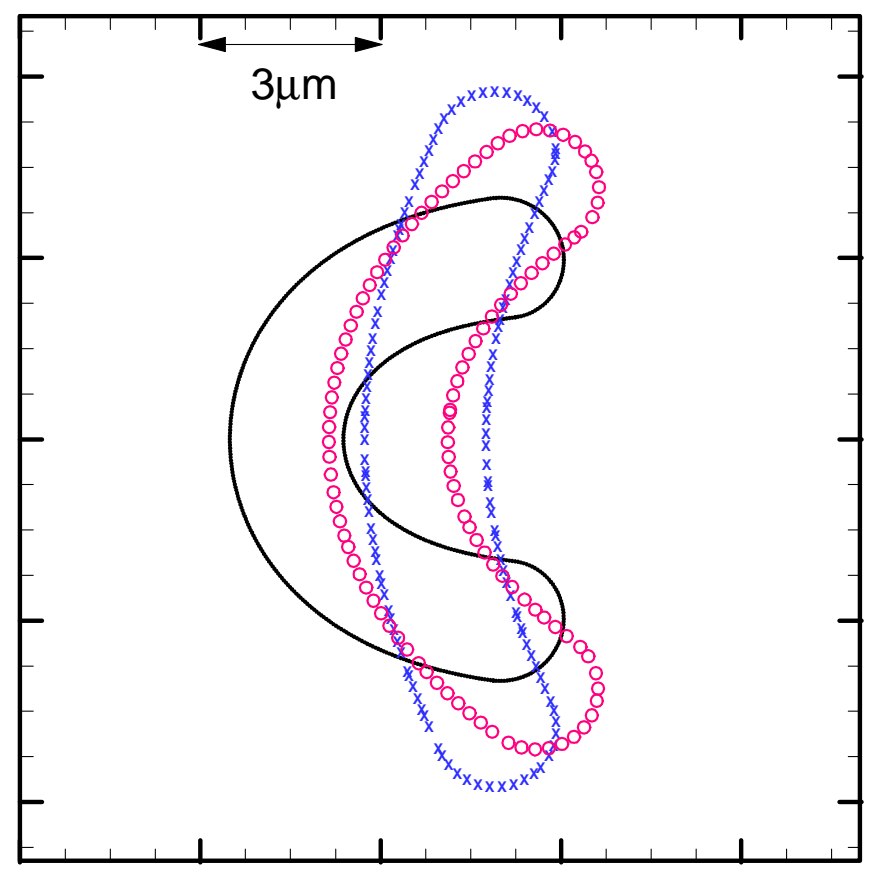

Figure 11 Equilibrium shape of a hyperelastic particle when $E=20 \mathrm{KV} / \mathrm{m}, G_{0}=25 \mathrm{~Pa}, \theta_{p 0}=90^{\circ}$ and $d=50 \mu \mathrm{m}$. Solid line, circles and crosses denote, respectively, the equilibrium particle shape when $\zeta_{p}=-50 \mathrm{mV}(t=16 \mathrm{~ms}),-25 \mathrm{mV}(t=32 \mathrm{~ms})$ and $-10 \mathrm{mV}(t=80 \mathrm{~ms})$. 


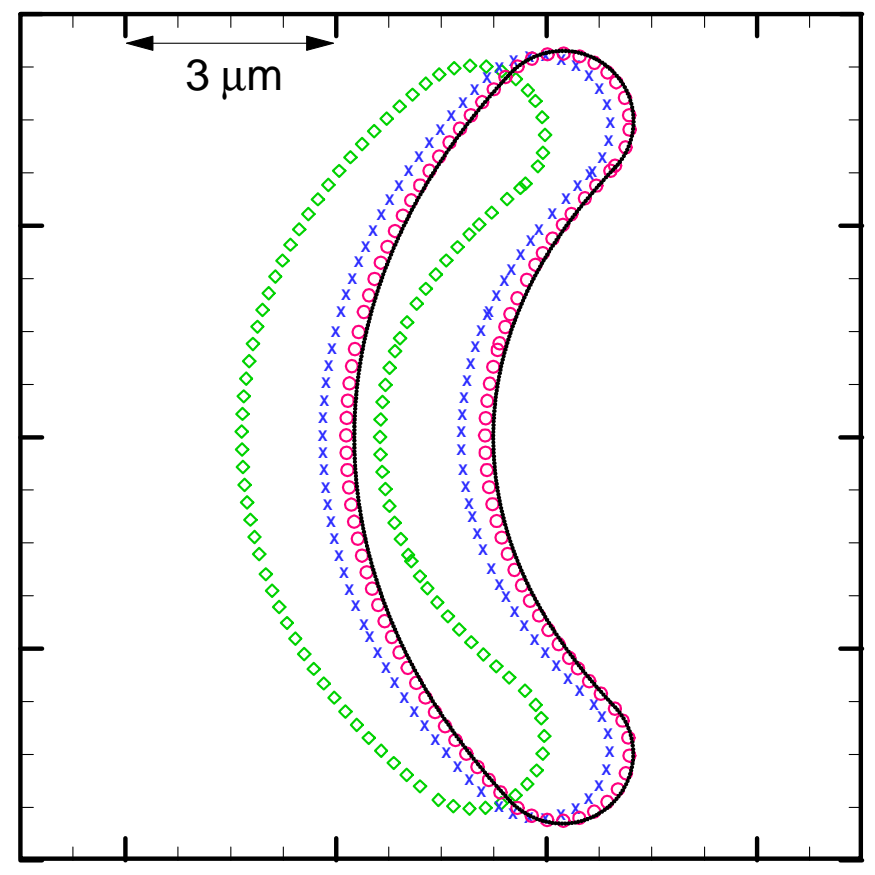

Figure 12 Equilibrium shape of a hyperelastic particle when $E=10 \mathrm{KV} / \mathrm{m}, G_{0}=25 \mathrm{~Pa}, \theta_{p 0}=90^{\circ}$ and $\zeta_{p}=-50 \mathrm{mV}$. Solid line, circles, crosses and diamonds denote, respectively, the equilibrium particle shape when $d=50,40,30$ and $20 \mu \mathrm{m}$. 